\title{
Uniform Resolvent Estimates for Magnetic Schrödinger Operators in a 2D Exterior Domain and their Applications to Related Evolution Equations
}

\author{
by
}

Kiyoshi Mochizuki and Hideo NaKazawa

\begin{abstract}
We consider the magnetic Schrödinger operator in an exterior domain $\Omega \subset \mathbb{R}^{2}$ with starshaped boundary with respect to the origin. We prove uniform resolvent estimates under suitable decay and smallness conditions on the magnetic field and external potential. The results are then used to obtain smoothing properties for the corresponding evolution equations.
\end{abstract}

2010 Mathematics Subject Classification: Primary 35Q40; Secondary 81Q10.

Keywords: magnetic Schrödinger operator, smoothing property.

\section{§1. Introduction and results}

Let $\Omega$ be an exterior domain in $\mathbb{R}^{2}$ with a star-shaped complement with respect to the origin and smooth boundary $\partial \Omega$. In this paper we consider in $\Omega$ the magnetic Schrödinger equation

$$
-\sum_{j=1}^{2}\left\{\partial_{j}+i b_{j}(x)\right\}^{2} u+c(x) u-\kappa^{2} u=f(x), \quad x \in \Omega,
$$

with Dirichlet boundary condition

$$
u(x, \kappa)=0, \quad x \in \partial \Omega .
$$

Communicated by H. Okamoto. Received November 19, 2013. Revised April 24, 2014, October 9, 2014, and November 17, 2014.

K. Mochizuki: Department of Mathematics, Chuo University, Kasuga, Bunnkyou, Tokyo 112-8551, Japan (Emeritus Tokyo Metropolitan University);

e-mail: mochizuk@math.chuo-u.ac.jp

H. Nakazawa: Department of Mathematics, Nippon Medical School, Kyonan-cho, Musashino, Tokyo 180-0023, Japan;

e-mail: nakazawa-hideo@nms.ac.jp 
Here $\partial_{j}=\partial / \partial x_{j}(j=1,2), i=\sqrt{-1}, \kappa \in \Pi_{ \pm}=\{\kappa \in \mathbb{C} ; \pm \operatorname{Re} \kappa>0, \operatorname{Im} \kappa>0\}$, $b_{j}(x)$ are real valued $C^{1}$-functions of $x \in \bar{\Omega}=\Omega \cup \partial \Omega, c(x)$ is a real valued continuous function of $x \in \bar{\Omega}$ and $f \in L^{2}(\Omega)$. Moreover, $b(x)=\left(b_{1}(x), b_{2}(x)\right)$ represents a magnetic potential. Thus the magnetic field is defined by its rotation, $\nabla \times b(x)=\partial_{1} b_{2}(x)-\partial_{2} b_{1}(x)$.

We set $\nabla=\left(\partial_{1}, \partial_{2}\right), \nabla_{b}=\nabla+i b(x), \Delta_{b}=\nabla_{b} \cdot \nabla_{b}, r=|x|, \tilde{x}=x / r$ and $\partial_{r}=\tilde{x} \cdot \nabla$. The inner product and norm of $L^{2}(\Omega)$ are defined by

$$
(f, g)=\int f(x) \overline{g(x)} d x \quad \text { and } \quad\|f\|=\sqrt{(f, f)} .
$$

Here $\int d x$ denotes integration over $\Omega$. Moreover, for $t>0$ we set

$$
\Omega_{t}=\{x \in \Omega ;|x|<t\} \quad \text { and } \quad S_{t}=\{x \in \Omega ;|x|=t\} .
$$

Now we define an operator $L$ acting in $L^{2}(\Omega)$ as follows:

$$
\left\{\begin{array}{l}
L u=-\Delta_{b} u+c(x) u \text { for } u \in \mathcal{D}(L), \\
\mathcal{D}(L)=\left\{u \in L^{2}(\Omega) \cap H_{\mathrm{loc}}^{2}(\bar{\Omega}) ;\left(-\Delta_{b}+c\right) u \in L^{2},\left.u\right|_{\partial \Omega}=0\right\} .
\end{array}\right.
$$

Here $H^{j}(\Omega)(j=1,2, \ldots)$ are the usual Sobolev spaces on $\Omega$ and $H_{\mathrm{loc}}^{2}(\bar{\Omega})$ is the space of functions that are $H^{2}$ on each compact set of $\bar{\Omega}$.

As is well known, if $c \in L^{2}(\Omega)$, then $L$ is a lower semibounded selfadjoint operator in $L^{2}(\Omega)$ (see e.g. Mochizuki [13]). Moreover, if

$$
\max \{|\nabla \times b(x)|,|c(x)|\}=o\left(r^{-1}\right) \quad \text { as } r \rightarrow \infty
$$

then the essential spectrum of $L$ fills the whole nonnegative half-line $[0, \infty)$ (see Aliev-Eyvazov [1]).

The selfadjointness of $L$ shows that its resolvent $R\left(\kappa^{2}\right)=\left(L-\kappa^{2}\right)^{-1}$ is defined for each $\kappa \in \Pi_{ \pm}$, and $u=R\left(\kappa^{2}\right) f$ gives a unique $L^{2}$-solution of the exterior problem (1.1), (1.2).

In the following we exclude the case $\Omega=\mathbb{R}^{2}$ and assume that

$$
\text { there exists } r_{0}>0 \text { such that } \min \{|x| ; x \in \partial \Omega\}>r_{0} .
$$

Now the main purpose of this paper is to show the following theorem for the solution $u=R\left(\kappa^{2}\right) f$.

Theorem 1. Assume that (A0) holds and

$$
\left\{|\nabla \times b(x)|^{2}+|c(x)|^{2}\right\}^{1 / 2} \leq \frac{\epsilon_{0}}{r^{2}\left(1+\log r / r_{0}\right)^{2}} \quad \text { in } \Omega,
$$

where $0<\epsilon_{0}<1 /(4 \sqrt{21})$ and we mean $\log r / r_{0}=\log \left(r / r_{0}\right)$. 
(i) For each $\kappa \in \Pi_{ \pm}$, we have

$$
\int(\operatorname{Im} \kappa r+1) \frac{|u|^{2}}{r^{2}\left(1+\log r / r_{0}\right)^{2}} d x \leq C_{1} \int r^{2}\left(1+\log r / r_{0}\right)^{2}|f|^{2} d x
$$

with $C_{1}=(2 \sqrt{21})^{2} /\left(1-\left(4 \sqrt{21} \epsilon_{0}\right)^{2}\right)$. Moreover, if we define the vector function

$$
\theta(x, \kappa)=\nabla_{b} u+\tilde{x}\left(\frac{1}{2 r}-i \kappa\right) u,
$$

then for each $\kappa \in \Pi_{ \pm}$,

$$
\int(\operatorname{Im} \kappa r+1) \frac{|\theta|^{2}}{\left(4+\log r / r_{0}\right)^{2}} d x \leq C_{2} \int r^{2}\left(1+\log r / r_{0}\right)^{2}|f|^{2} d x
$$

where $C_{2}=C_{2}\left(\epsilon_{0}\right) \leq 2+2\left(1+\epsilon_{0}^{2}\right) C_{1}$.

(ii) Let $\mu(r)$ be a smooth, positive $L^{1}$-function of $r \geq r_{0}$ satisfying also

$$
2 r \mu^{\prime}(r) \leq \mu(r) \quad \text { and } \quad \mu(r) \leq \frac{C_{3}}{\left(4+\log r / r_{0}\right)^{2}}
$$

for some $C_{3}>0$. Then for each $\kappa \in \Pi_{ \pm}$,

$$
\int \mu(r)\left\{\left|\nabla_{b} u\right|^{2}+|\kappa u|^{2}\right\} d x \leq C_{4} \int\left\{r^{2}\left(1+\log r / r_{0}\right)^{2}+\mu(r)^{-1}\right\}|f(x)|^{2} d x
$$

with $C_{4}=\max \left\{2 C_{3} C_{2}, 4\|\mu\|_{L^{1}}^{2}\right\}$, where $\|\mu\|_{L^{1}}=\int_{r_{0}}^{\infty} \mu(t) d t$.

Remark 1. (1) The function $\theta(x, \kappa)$ is used to define radiation conditions for the solution $u(x, \kappa)$ (see [13]), and (1.7) asserts their uniform estimates in $\kappa \in \Pi_{ \pm}$.

(2) We can choose $\mu(r)=r^{-2}\left(1+\log r / r_{0}\right)^{-2}$. In this case (1.8) holds with $C_{3}=\left(4 / r_{0}\right)^{2}$.

Remark 2. Theorem 1(i) implies that the operator $L$ is nonnegative and 0 is not an eigenvalue of $L$. The above two assertions can also be proved directly. Note that

$$
\liminf _{t \rightarrow \infty} \operatorname{Re} \int_{S_{t}}(\tilde{x} \cdot \nabla u) \bar{u} d S \leq 0
$$

for $u \in \mathcal{D}(L)$ (see [15]). Then a direct proof follows from the identity

$\operatorname{Re} \int_{\Omega_{t}}\left\{-\nabla_{b} \cdot \nabla_{b} u+c(x) u\right\} \bar{u} d x=\int_{\Omega_{t}}\left\{\left|\nabla_{b} u\right|^{2}+c(x)|u|^{2}\right\} d x-\operatorname{Re} \int_{S_{t}}(\tilde{x} \cdot \nabla u) \bar{u} d S$ combined with the modified Hardy inequality (Lemma 4 ) of $\S 3$ and the requirement $c(x) \geq-\epsilon_{0} r^{-2}\left(1+\log r / r_{0}\right)^{-2}$ with $\epsilon_{0}<1 / 4$. 
As a corollary of Theorem 1, we are able to obtain space-time weighted estimates (smoothing properties, cf. Kato [8]) for the Schrödinger evolution equation

$$
i \frac{\partial u}{\partial t}-L u=0, \quad u(0)=f \in L^{2}(\Omega),
$$

and for the relativistic Schrödinger evolution equation

$$
i \frac{\partial u}{\partial t}-\sqrt{L+m^{2}} u=0, \quad u(0)=f \in L^{2}(\Omega),
$$

with $m \geq 0$. Note that the smoothing effects for (1.11) give those for the KleinGordon equation $(m>0)$ or the wave equation $(m=0)$ in the energy space.

Theorem 2. Assume that (A0) and (A1) hold.

(i) The solution operator $e^{-i t L}$ to (1.10) satisfies

$$
\begin{aligned}
& \left|\int_{0}^{ \pm \infty}\left\|r^{-1}\left(1+\log r / r_{0}\right)^{-1} \int_{0}^{t} e^{-i(t-\tau) L} h(\tau) d \tau\right\|^{2} d t\right| \\
& \leq C_{1}\left|\int_{0}^{ \pm \infty}\left\|r\left(1+\log r / r_{0}\right) h(t)\right\|^{2} d t\right|
\end{aligned}
$$

for every $h(t)$ satisfying $r\left(1+\log r / r_{0}\right) h(t) \in L^{2}(\mathbb{R} \times \Omega)$, and

$$
\left|\int_{0}^{ \pm \infty}\left\|r^{-1}\left(1+\log r / r_{0}\right)^{-1} e^{-i t L} f\right\|^{2} d t\right| \leq 2 \sqrt{C_{1}}\|f\|^{2}
$$

for $f \in L^{2}(\Omega)$. Here $\|\cdot\|$ means the norm of $L^{2}(\Omega)$.

(ii) The solution operator $e^{-i t \sqrt{L+m}}$ to (1.11) satisfies

$$
\left|\int_{0}^{ \pm \infty}\left\|r^{-1}\left(1+\log r / r_{0}\right)^{-1} e^{-i t \sqrt{L+m^{2}}} f\right\|^{2} d t\right| \leq 8 \sqrt{m^{2} C_{1}+2 C_{4}}\|f\|^{2}
$$

for $f \in L^{2}(\Omega)$. Here to determine $C_{4}$ we have chosen $\mu(r)=r^{-2}\left(1+\log r / r_{0}\right)^{-2}$.

For space dimension $n \geq 3$, Theorems 1 and 2 have been proved by Mochizuki [14], [15] with weight $r^{-1}\left(1+\log r / r_{0}\right)^{-1}$ in (A1) replaced by $r^{-1}$ for problems in both an exterior domain and the whole space $\mathbb{R}^{n}$. In case $n=2$ some partial results have been obtained by Nakazawa [14], but without the resolvent estimate being given clearly in the duality form with weight $r^{-1}\left(1+\log r / r_{0}\right)^{-1}$. To show (1.5) it is crucial to obtain a Hardy type inequality corresponding to the functions $\theta(x, \kappa)$ (see Proposition 3 and Lemma 3 below). In case $n \geq 3$ the inequality is not so far from the original Hardy inequality, and reads (see [15, Lemma 9])

$$
\int \frac{(n-2)^{2}}{4 r^{2}}|u|^{2} d x \leq \int\left\{|\tilde{x} \cdot \theta|^{2}+\frac{(n-1)(n-3)}{4 r^{2}}|u|^{2}\right\} d x .
$$


In [15] the proof of Theorem 1(i) is done by combining this with another inequality for the solution $u=R\left(\kappa^{2}\right) f$ which corresponds to Lemma 2 below. In case $n=2$ the above inequality does not work since the left side vanishes identically. Moreover, the definiteness of the integrand $|\tilde{x} \cdot \theta|^{2}-\frac{1}{4 r^{2}}|u|^{2}$ is not guaranteed. So, we need a delicate calculation to modify it. As a result, the function

$$
\eta(r)=\frac{1}{2 r\left(1+\log r / r_{0}\right)}
$$

is adopted to be a suitable weight satisfying the inequality

$$
\int \eta^{2}|u|^{2} d x \leq C \int\left(\operatorname{Im} \kappa r+\frac{1}{2}-\tilde{\epsilon} r^{2} \eta^{2}\right)\left\{|\tilde{x} \cdot \theta|^{2}-\frac{1}{4 r^{2}}|u|^{2}\right\} d x
$$

with $C=C(\tilde{\epsilon})>0$ for small $\tilde{\epsilon}>0$ (see Lemmas 2 and 3). The above weight is the same as the one for the Hardy inequality in an exterior domain of $\mathbb{R}^{2}$ (Remark 3 of $\S 3$ ). Note that the standard Hardy inequality does not in general hold in the whole space $\mathbb{R}^{2}$ (see e.g. Solomyak [19], Kobayashi-Misawa [10]).

Theorem 1(i) shows the $L^{2}$-smoothness of $r^{-1}\left(1+\log r / r_{0}\right)^{-1}$. Apart from [14], [15], the corresponding results have been studied by Kato-Yajima [9] (see also Kuroda [11], Watanabe [20]), where the operator in question is the Laplace operator $L_{0}=-\Delta$ in $\mathbb{R}^{n}(n \geq 3)$. Fourier transformation methods are used there, and the results are summarized for $n \geq 2$ as follows:

$$
\left\|r^{-\alpha} L_{0}^{\beta}\left(L_{0}-\kappa^{2}\right)^{-1} r^{-\alpha} f\right\| \leq C|\kappa|^{2(\alpha+\beta-1)}\|f\|,
$$

where $\alpha \in(1 / 2, n / 2)$ and $\beta \in[-\alpha, 1-\alpha]$ (Yafaev [21, Proposition 7.1.11]). Note that the right side is never bounded when $n=2$ and $\beta=0$. More explicitly, the asymptotic expansions of the resolvent $\left(-\Delta-\kappa^{2}\right)^{-1}$ as $\kappa \rightarrow 0$ have been well studied in suitable weighted $L^{2}$-spaces (see Murata [16], Yajima [23], JensenNenciu [7]), and in case $n=2$ a logarithmic singularity $\log \kappa$ appears in the leading term. Thus, the uniform estimates of Theorem 1(i) are not expected to hold for two-dimensional whole space problems.

We should mention here that when a suitable non-trivial magnetic field is introduced, one can expect some improvement of the above situation. In fact, the Hardy inequality in Remark 3 is known to hold without the unpleasant logarithmic factor for such a magnetic Laplacian $-\Delta_{b}$ in the whole $\mathbb{R}^{2}$ (Laptev-Weidl [12]). So, there is some possibility to develop a new low-energy estimate of $\left(-\Delta_{b}-\kappa^{2}\right)^{-1}$. In this paper, however, we do not enter into this problem. Our assumption (A1) requires only the smallness of the magnetic field, and it does not exclude the zero magnetic field $\nabla \times b(x) \equiv 0$.

For the exterior problem, Fourier transformation methods are not applicable, and in general it becomes difficult to know clearly the $\kappa$ dependence of the resol- 
vent kernel. It seems that, especially when $n=2$, there are no results showing the resolvent expansions around zero and/or high energies. Resolvent estimates are mainly developed in connection with the study of the principle of limiting absorption. So, the results are restricted to $\kappa$ in a compact domain in $\Pi_{ \pm} \backslash\{0\}$ (e.g., [13], Reed-Simon [18]).

Results similar to Theorem 2 have been studied by many authors in connection with local smoothing properties (see, e.g., Kato-Yajima [9], Yajima [22], Ben-Artzi [2], Cuccagna-Schirmer [3], D'Ancona-Fanelli [4], Erdogan-GoldbergSchlag [5] and Georgiev-Stefanov-Tarulli [6]). Note that these works are also restricted to the case of space dimension $n \geq 3$, and the vector potential $b(x)$ itself is required to be small and to decay sufficiently fast (the smallness is not required in [5]). On the other hand, no such requirement appears in our case, but the smallness of the magnetic field $\nabla \times b(x)$ is required. It seems difficult to remove it without any decay conditions on $b(x)$.

The rest of this paper is organized as follows. Theorems 1 and 2 are proved in $\S 3$ and $\S 4$, respectively. In $\S 2$ we prepare three propositions which are essential in proving Theorem 1.

\section{$\S 2$. Preliminaries}

The first two propositions are quadratic identities for a solution $u$ of problem (1.1), $(1.2)$.

We multiply (1.1) by $-\overline{i \kappa u}$ to obtain

$$
\nabla \cdot\left\{\left(\nabla_{b} u\right) \overline{i \kappa u}\right\}-\overline{i \kappa}\left\{\left|\nabla_{b} u\right|^{2}+c(x)|u|^{2}-\kappa^{2}|u|^{2}\right\}=-f \overline{i \kappa u}
$$

and integrate the real part of this equation over $\Omega_{t}\left(t>r_{0}\right)$. By the boundary condition (1.2),

$$
\begin{aligned}
\operatorname{Re} \int_{\Omega_{t}} \nabla \cdot\left\{\left(\nabla_{b} u\right) \overline{i \kappa u}\right\} d x & =\operatorname{Re} \int_{S_{t}}\left(\tilde{x} \cdot \nabla_{b} u\right) \overline{i \kappa u} d S \\
& =\frac{1}{2} \int_{S_{t}}\left\{-\left|\nabla_{b} u-i \kappa \tilde{x} u\right|^{2}+\left|\nabla_{b} u\right|^{2}+|\kappa u|^{2}\right\} d S
\end{aligned}
$$

and it follows that

$$
\begin{aligned}
& \frac{1}{2} \int_{S_{t}}\left\{-\left|\nabla_{b} u-i \kappa u\right|^{2}+\left|\nabla_{b} u\right|^{2}+|\kappa u|^{2}\right\} d S \\
& \quad+\operatorname{Im} \kappa \int_{\Omega_{t}}\left(\left|\nabla_{b} u\right|^{2}+c|u|^{2}+|\kappa u|^{2}\right) d x=-\operatorname{Re} \int_{\Omega_{t}} f \overline{i \kappa u} d x
\end{aligned}
$$


Here

$-\left|\nabla_{b} u-i \kappa \tilde{x} u\right|^{2}=-\left|\nabla_{b} u+\tilde{x}\left(\frac{1}{2 r}-i \kappa\right) u\right|^{2}+\frac{1}{4 r^{2}}|u|^{2}+\operatorname{Im} \kappa \frac{1}{r}|u|^{2}+\nabla \cdot\left\{\frac{1}{2 r} \tilde{x}|u|^{2}\right\}$.

The following proposition is a direct consequence of (2.2) multiplied by $\mu(t)$ and integrated over $\left(r_{0}, \infty\right)$.

Proposition 1. Let $u=R\left(\kappa^{2}\right) f$. Then

$$
\begin{aligned}
\frac{1}{2} \int\left\{\left(\operatorname{Im} \kappa \frac{\mu}{r}+\right.\right. & \left.\left.\frac{\mu}{4 r^{2}}-\frac{\mu^{\prime}}{2 r}\right)|u|^{2}+\mu\left(\left|\nabla_{b} u\right|^{2}+|\kappa u|^{2}\right)\right\} d x \\
& +\operatorname{Im} \kappa \int_{r_{0}}^{\infty} \mu(t) d t \int_{\Omega_{t}}\left\{\left|\nabla_{b} u\right|^{2}+c(x)|u|^{2}+|\kappa u|^{2}\right\} d x \\
& =\frac{1}{2} \int \mu|\theta|^{2} d x-\operatorname{Re} \int_{r_{0}}^{\infty} \mu(t) d t \int_{\Omega_{t}} f \overline{i \kappa u} d x,
\end{aligned}
$$

where $\theta=\nabla_{b} u+\tilde{x}\left(\frac{1}{2 r}-i \kappa\right) u$.

Next we set $v=e^{-i \kappa r} r^{1 / 2} u, g=e^{-i \kappa r} r^{1 / 2} f$ and rewrite (1.1) as follows:

$$
-\nabla_{b} \cdot \nabla_{b} v+\left(-2 i \kappa+\frac{1}{r}\right) \tilde{x} \cdot \nabla_{b} v+\left(c-\frac{1}{4 r^{2}}\right) v=g
$$

Let $\phi=\phi(r)=e^{-2 \operatorname{Im} \kappa r} r^{-1} \varphi(r)$, where $\varphi(r)$ is a smooth, positive function of $r>0$. We multiply $(2.3)$ by $\phi \overline{\tilde{x} \cdot \nabla_{b} v}$. Note that

$$
\begin{aligned}
-\nabla_{b} \cdot \nabla_{b} v \phi \overline{\tilde{x} \cdot \nabla_{b} v}= & -\nabla \cdot\left\{\nabla_{b} v \phi \overline{\tilde{x} \cdot \nabla_{b} v}\right\}+\left(\nabla \phi \cdot \nabla_{b} v\right) \overline{\tilde{x} \cdot \nabla_{b} v} \\
& +\phi \nabla_{b} v \cdot \overline{\nabla_{b}\left(\tilde{x} \cdot \nabla_{b} v\right)} .
\end{aligned}
$$

In the last term of the right side we use the identity

$$
\begin{aligned}
\left(\partial_{j}+i b_{j}\right)\left\{\tilde{x}_{k}\left(\partial_{k}+i b_{k}\right) v\right\}= & \tilde{x}_{k}\left(\partial_{k}+i b_{k}\right)\left\{\left(\partial_{j}+i b_{j}\right) v\right\} \\
& +\frac{\delta_{j k}-\tilde{x}_{j} \tilde{x}_{k}}{r}\left(\partial_{k}+i b_{k}\right) v+i \tilde{x}_{k}\left(\partial_{j} b_{k}-\partial_{k} b_{j}\right) v
\end{aligned}
$$

Then it follows that

$$
\begin{aligned}
\operatorname{Re}\left\{\phi \nabla_{b} v \cdot \overline{\nabla_{b}\left(\tilde{x} \cdot \nabla_{b} v\right)}\right\}= & \frac{1}{2} \nabla \cdot\left\{\phi \tilde{x}\left|\nabla_{b} v\right|^{2}\right\}-\left(\frac{\phi^{\prime}}{2}+\frac{\phi}{2 r}\right)\left|\nabla_{b} v\right|^{2} \\
& +\frac{\phi}{r}\left(\left|\nabla_{b} v\right|^{2}-\left|\tilde{x} \cdot \nabla_{b} v\right|^{2}\right)-\phi \operatorname{Re}\left\{\left(\tilde{x} \times \nabla_{b} v\right) \overline{(\nabla \times i b) v}\right\},
\end{aligned}
$$

and we finally obtain 


$$
\begin{aligned}
& -\operatorname{Re} \nabla \cdot\left\{\left(\phi \nabla_{b} v\right) \overline{\tilde{x} \cdot \nabla_{b} v}\right\}+\phi^{\prime}\left|\tilde{x} \cdot \nabla_{b} v\right|^{2}+\frac{1}{2} \nabla \cdot\left\{\phi \tilde{x}\left|\nabla_{b} v\right|^{2}\right\} \\
& -\left(\frac{\phi^{\prime}}{2}+\frac{\phi}{2 r}\right)\left|\nabla_{b} v\right|^{2}+\frac{\phi}{r}\left(\left|\nabla_{b} v\right|^{2}-\left|\tilde{x} \cdot \nabla_{b} v\right|^{2}\right) \\
& -\phi \operatorname{Re}\left\{\left(\tilde{x} \times \nabla_{b} v\right) \overline{(\nabla \times i b) v}\right\}+\phi\left(2 \operatorname{Im} \kappa+\frac{1}{r}\right)\left|\tilde{x} \cdot \nabla_{b} v\right|^{2} \\
& +\operatorname{Re} \phi\left\{\left(c-\frac{1}{4 r^{2}}\right) v \overline{\tilde{x} \cdot \nabla_{b} v}\right\}=\operatorname{Re} \phi\left\{g \overline{\tilde{x} \cdot \nabla_{b} v}\right\} .
\end{aligned}
$$

Note here $\nabla_{b} v=e^{-i \kappa r} r^{1 / 2} \theta$, and

$$
\begin{aligned}
& \phi^{\prime}(r)=\phi(r)\left(-2 \operatorname{Im} \kappa-\frac{1}{r}+\frac{\varphi^{\prime}}{\varphi}\right) \\
& -\operatorname{Re}\left\{\frac{\phi}{4 r^{2}} v \overline{\tilde{x} \cdot \nabla_{b} v}\right\}=-\frac{1}{2} \nabla \cdot\left\{\frac{\varphi}{4 r^{2}} \tilde{x}|u|^{2}\right\}+\varphi\left(-\operatorname{Im} \kappa-\frac{1}{r}+\frac{\varphi^{\prime}}{2 \varphi}\right) \frac{1}{4 r^{2}}|u|^{2} .
\end{aligned}
$$

Substituting these relations to (2.4) and integrating both sides over $\Omega_{t}$, we obtain

Proposition 2. Let $u=R\left(\kappa^{2}\right) f$. Then for $t$ large,

$$
\begin{gathered}
-\int_{S_{t}} \varphi\left\{|\tilde{x} \cdot \theta|^{2}-\frac{1}{2}|\theta|^{2}+\frac{1}{8 r^{2}}|u|^{2}\right\} d S-\int_{\partial \Omega} \varphi\left\{(\nu \cdot \nabla u)(\tilde{x} \cdot \overline{\nabla u})-\frac{1}{2}(\nu \cdot \tilde{x})|\nabla u|^{2}\right\} d S \\
+\int_{\Omega_{t}} \varphi\left[-\left(\frac{1}{r}-\frac{\varphi^{\prime}}{\varphi}\right)|\tilde{x} \cdot \theta|^{2}+\left(\operatorname{Im} \kappa+\frac{1}{r}-\frac{\varphi^{\prime}}{2 \varphi}\right)\left\{|\theta|^{2}-\frac{1}{4 r^{2}}|u|^{2}\right\}\right. \\
+\operatorname{Re}\{-(\nabla \times i b) u \overline{\tilde{x} \times \theta}+c u \tilde{\tilde{x} \cdot \theta}\}] d x=\operatorname{Re} \int_{\Omega_{t}} \varphi f \overline{\tilde{x} \cdot \theta} d x
\end{gathered}
$$

where $\nu=\nu(x)$ is the outer unit normal to the boundary $\partial \Omega$.

Let $H_{0, b}^{1}=H_{0, b}^{1}(\Omega)$ denote the completion of $C_{0}^{\infty}(\Omega)$ with respect to the norm

$$
\|u\|_{H_{b}^{1}}^{2}=\int\left\{|u(x)|^{2}+\left|\nabla_{b} u(x)\right|^{2}\right\} d x .
$$

Obviously $\mathcal{D}(L) \subset H_{0, b}^{1}$.

Proposition 3. Let $\eta=\eta(r)$ and $\xi=\xi(r)$ be smooth, positive functions of $r \geq 0$, and let $t$ be large. Then for each $u \in H_{0, b}^{1}$,

$$
\begin{aligned}
\int_{\Omega_{t}} \xi\left\{|\tilde{x} \cdot \theta|^{2}-\frac{1}{4 r^{2}}|u|^{2}\right\} & d x=\int_{\Omega_{t}} \xi\left|\tilde{x} \cdot \nabla_{b} u-i \kappa u-\eta u\right|^{2} d x \\
& +\int_{S_{t}} \xi\left(\frac{1}{2 r}+\eta\right)|u|^{2} d S-\int_{\Omega_{t}} \xi^{\prime}\left(\frac{1}{2 r}+\eta\right)|u|^{2} d x \\
& +\int_{\Omega_{t}} \xi\left\{2 \operatorname{Im} \kappa\left(\frac{1}{2 r}+\eta\right)|u|^{2}-\left(\frac{1}{r} \eta+\eta^{\prime}+\eta^{2}\right)|u|^{2}\right\} d x .
\end{aligned}
$$


Proof. Note that

$$
\begin{aligned}
|\tilde{x} \cdot \theta|^{2}= & \left|\tilde{x} \cdot \nabla_{b} u+\frac{1}{2 r} u-i \kappa u-\eta u+\eta u\right|^{2} \\
= & \left|\tilde{x} \cdot \nabla_{b} u-i \kappa u-\eta u\right|^{2}+\nabla \cdot\left\{\tilde{x}\left(\frac{1}{2 r}+\eta\right)|u|^{2}\right\} \\
& +2 \operatorname{Im} \kappa\left(\frac{1}{2 r}+\eta\right)|u|^{2}+\frac{1}{4 r^{2}}|u|^{2}-\left(\frac{1}{r} \eta+\eta^{\prime}+\eta^{2}\right)|u|^{2} .
\end{aligned}
$$

Multiplying by $\xi(r)$ and integrating over $\Omega_{t}$, we deduce the desired identity since $\left.u\right|_{\partial \Omega}=0$.

\section{§3. Proof of Theorem 1}

In this section we shall prove Theorem 1 by a series of lemmas for the solution $u=R\left(\kappa^{2}\right) f$ of $(1.1),(1.2)$.

Lemma 1. Let $\varphi=\varphi(r)$ be a positive increasing function of $r>r_{0}$ satisfying

$$
\frac{\varphi^{\prime}(r)}{\varphi(r)} \leq \frac{1}{r}
$$

Then

$$
\begin{aligned}
\int \varphi\left\{\left(\operatorname{Im} \kappa+\frac{\varphi^{\prime}}{2 \varphi}\right)|\theta|^{2}-(\operatorname{Im}\right. & \left.\left.\kappa+\frac{1}{r}-\frac{\varphi^{\prime}}{2 \varphi}\right) \frac{1}{4 r^{2}}|u|^{2}\right\} d x \\
& \leq \int \varphi\left\{|f|+\left(|\nabla \times b|^{2}+|c|^{2}\right)^{1 / 2}|u|\right\}|\theta| d x .
\end{aligned}
$$

Proof. In Proposition 2 we let $t \rightarrow \infty$. Then since $\varphi(r)=O(r)$ as $r \rightarrow \infty$, we have

$$
\liminf _{t \rightarrow \infty} \int_{S_{t}} \varphi\left\{|\tilde{x} \cdot \theta|^{2}-\frac{1}{2}|\theta|^{2}+\frac{1}{8 r^{2}}|u|^{2}\right\} d S=0 .
$$

On the other hand, since $\nabla u=(\nu \cdot \nabla u) \nu$ on $\partial \Omega$, the starshapedness of $\partial \Omega$ implies that

$$
\int_{\partial \Omega} \varphi\left\{-(\nu \cdot \nabla u)(\tilde{x} \cdot \overline{\nabla u})+\frac{1}{2}(\nu \cdot \tilde{x})|\nabla u|^{2}\right\} d S=-\frac{1}{2} \int_{\partial \Omega} \varphi(\nu \cdot \tilde{x})|\nu \cdot \nabla u|^{2} d S \geq 0 .
$$

Thus, the desired inequality follows if we note that

$$
\begin{aligned}
& \left(\frac{1}{r}-\frac{\varphi^{\prime}}{\varphi}\right)\left\{|\theta|^{2}-|\tilde{x} \cdot \theta|^{2}\right\} \geq 0, \\
& |-(\nabla \times i b) u \overline{\tilde{x} \times \theta}+c u \overline{\tilde{x} \cdot \theta}| \leq\left(|\nabla \times b|^{2}+|c|^{2}\right)^{1 / 2}|u||\theta| .
\end{aligned}
$$


Lemma 2. For any $\epsilon>0$,

$$
\begin{aligned}
\int(\operatorname{Im} \kappa r+ & \left.\frac{1}{2}-\frac{2 \epsilon}{\left(1+\log r / r_{0}\right)^{2}}\right)\left\{|\theta|^{2}-\frac{1}{4 r^{2}}|u|^{2}\right\} d x \\
& \leq \frac{1}{4 \epsilon} \int r^{2}\left(1+\log r / r_{0}\right)^{2}|f|^{2} d x+\left(\frac{\epsilon_{0}^{2}}{\epsilon}+2 \epsilon\right) \int \frac{|u|^{2}}{4 r^{2}\left(1+\log r / r_{0}\right)^{2}} d x .
\end{aligned}
$$

Proof. We choose $\varphi=r$ in Lemma 1 and use the Schwarz inequality. Then noting (A1), we have

$$
\begin{aligned}
\int(\operatorname{Im} \kappa r+ & \left.\frac{1}{2}\right)\left\{|\theta|^{2}-\frac{1}{4 r^{2}}|u|^{2}\right\} d x \\
\leq & \int\left\{\frac{r^{2}\left(1+\log r / r_{0}\right)^{2}|f|^{2}}{4 \epsilon}+\frac{\epsilon_{0}^{2}|u|^{2}}{4 \epsilon r^{2}\left(1+\log r / r_{0}\right)^{2}}\right\} d x \\
& +\int \frac{2 \epsilon}{\left(1+\log r / r_{0}\right)^{2}}\left\{|\theta|^{2}-\frac{1}{4 r^{2}}|u|^{2}\right\} d x+\int \frac{2 \epsilon|u|^{2}}{4 r^{2}\left(1+\log r / r_{0}\right)^{2}} d x
\end{aligned}
$$

which proves the lemma.

Lemma 3. Let $u \in H_{0, b}^{1}$. Then for each $0<\epsilon<1 / 38$,

$$
\begin{aligned}
\int(\operatorname{Im} \kappa r+ & \left.\frac{1}{2}-19 \epsilon\right) \frac{|u|^{2}}{4 r^{2}\left(1+\log r / r_{0}\right)^{2}} d x \\
& \leq \int\left(\operatorname{Im} \kappa r+\frac{1}{2}-\frac{2 \epsilon}{\left(1+\log r / r_{0}\right)^{2}}\right)\left\{|\tilde{x} \cdot \theta|^{2}-\frac{1}{4 r^{2}}|u|^{2}\right\} d x .
\end{aligned}
$$

Proof. We choose

$$
\eta(r)=\frac{1}{2 r\left(1+\log r / r_{0}\right)}, \quad \xi(r)=\operatorname{Im} \kappa r+\frac{1}{2}-\frac{2 \epsilon}{\left(1+\log r / r_{0}\right)^{2}}
$$

in Proposition 3. Obviously

$$
\liminf _{t \rightarrow \infty} \int_{S_{t}} \xi\left(\frac{1}{2 r}+\eta\right)|u|^{2} d S=0
$$

Moreover, by definition $\xi(r)>0$ when $r>r_{0}$ and

$$
\frac{1}{r} \eta+\eta^{\prime}+\eta^{2}=\frac{-1}{4 r^{2}\left(1+\log r / r_{0}\right)^{2}} .
$$

Thus, the identity of Proposition 3 implies 


$$
\begin{aligned}
\int(\operatorname{Im} \kappa r & \left.+\frac{1}{2}-\frac{2 \epsilon}{\left(1+\log r / r_{0}\right)^{2}}\right)\left\{|\tilde{x} \cdot \theta|^{2}-\frac{1}{4 r^{2}}|u|^{2}\right\} d x \\
\geq & \int\left(\operatorname{Im} \kappa r+\frac{1}{2}-\frac{2 \epsilon}{\left(1+\log r / r_{0}\right)^{2}}\right) \\
& -\int\left(\operatorname{Im} \kappa+\frac{4 \epsilon}{r\left(1+\log r / r_{0}\right)^{3}}\right)\left(\frac{1}{2 r}+\eta\right)|u|^{2} d x \\
= & \int\left(\operatorname{Im} \kappa r+\frac{1}{2}-\frac{2 \epsilon}{\left(1+\log r / r_{0}\right)^{2}}\right) \frac{|u|^{2}}{4 r^{2}\left(1+\log r / r_{0}\right)^{2}} d x \\
& +\int\left(2(\operatorname{Im} \kappa)^{2} r-\frac{4 \operatorname{Im} \kappa \epsilon}{\left(1+\log r / r_{0}\right)^{2}}-\frac{4 \epsilon}{r\left(1+\log r / r_{0}\right)^{3}}\right)\left(\frac{1}{2 r}+\eta\right)|u|^{2} d x
\end{aligned}
$$

Since

$$
2(\operatorname{Im} \kappa)^{2} r-\frac{4 \operatorname{Im} \kappa \epsilon}{\left(1+\log r / r_{0}\right)^{2}} \geq \frac{-2 \epsilon^{2}}{r\left(1+\log r / r_{0}\right)^{4}}
$$

and $0<1 / 2 r+\eta \leq 1 / r$, noting

$$
\frac{1}{2}-2 \epsilon-8 \epsilon^{2}-16 \epsilon \geq \frac{1}{2}-19 \epsilon,
$$

we deduce the desired inequality.

Proof of Theorem 1(i). Combining Lemmas 2 and 3 we have, for $0<\epsilon<1 / 38$,

$$
\begin{aligned}
& \int\left(\operatorname{Im} \kappa r+\frac{1}{2}-19 \epsilon\right) \frac{|u|^{2}}{4 r^{2}\left(1+\log r / r_{0}\right)^{2}} d x \\
& \quad \leq \frac{1}{4 \epsilon} \int r^{2}\left(1+\log r / r_{0}\right)^{2}|f|^{2} d x+\left(\frac{\epsilon_{0}^{2}}{\epsilon}+2 \epsilon\right) \int \frac{|u|^{2}}{4 r^{2}\left(1+\log r / r_{0}\right)^{2}} d x .
\end{aligned}
$$

Here we require $\epsilon$ further to satisfy

$$
\frac{1}{2}-19 \epsilon-\frac{\epsilon_{0}^{2}}{\epsilon}-2 \epsilon>0
$$

or equivalently

$$
\epsilon_{0}^{2}<\epsilon\left(\frac{1}{2}-21 \epsilon\right)=-21\left(\epsilon-\frac{1}{84}\right)^{2}+\frac{1}{336} .
$$

This is possible if $\epsilon_{0}<1 /(4 \sqrt{21})$. In this case, we can choose $\epsilon=1 / 84$ in (3.3).

Then since

$$
\frac{1}{4 \epsilon}\left(\frac{1}{2}-21 \epsilon-\frac{\epsilon_{0}^{2}}{4 \epsilon}\right)^{-1}=\frac{(2 \sqrt{21})^{2}}{1-\left(4 \sqrt{21} \epsilon_{0}\right)^{2}},
$$

the desired inequality (1.5) follows from (3.2). 
To show (1.7) we return to the inequality of Lemma 1 choosing $\varphi=$ $r /\left(4+\log r / r_{0}\right)^{2}$. Concerning its left side, it follows from (1.5) that

$$
\begin{aligned}
\int \varphi\left(\operatorname{Im} \kappa+\frac{1}{r}-\frac{\varphi^{\prime}}{2 \varphi}\right) \frac{|u|^{2}}{4 r^{2}} d x & \leq \int(\operatorname{Im} \kappa r+1) \frac{|u|^{2}}{4 r^{2}\left(1+\log r / r_{0}\right)^{2}} d x \\
& \leq \frac{1}{4} C_{1} \int r^{2}\left(1+\log r / r_{0}\right)^{2}|f|^{2} d x
\end{aligned}
$$

Moreover, since $1 / r-\varphi^{\prime} / \varphi \geq 0$ and

$$
\varphi\left(\operatorname{Im} \kappa+\frac{\varphi^{\prime}}{2 \varphi}\right)=\varphi\left(\operatorname{Im} \kappa+\frac{1}{2 r}-\frac{1}{r\left(4+\log r / r_{0}\right)}\right) \geq \varphi\left(\operatorname{Im} \kappa+\frac{1}{4 r}\right)
$$

it follows that

$$
\int \varphi\left(\operatorname{Im} \kappa+\frac{\varphi^{\prime}}{2 \varphi}\right)|\theta|^{2} d x \geq \int\left(\operatorname{Im} \kappa r+\frac{1}{4}\right) \frac{|\theta|^{2}}{\left(4+\log r / r_{0}\right)^{2}} d x .
$$

On the other hand, by the Schwarz inequality the right side of the inequality of Lemma 1 is estimated as

$$
\begin{aligned}
& \int \frac{r}{\left(4+\log r / r_{0}\right)^{2}}\left\{|f|+\left(|\nabla \times b|^{2}+|c|^{2}\right)^{1 / 2}|u|\right\}|\theta| d x \\
& \leq \frac{1}{4 \epsilon} \frac{1}{4^{2}} \int\left\{r^{2}|f|^{2}+\epsilon_{0}^{2} \frac{|u|^{2}}{r^{2}\left(1+\log r / r_{0}\right)^{4}}\right\} d x+2 \epsilon \int \frac{|\theta|^{2}}{\left(4+\log r / r_{0}\right)^{2}} d x
\end{aligned}
$$

Thus, applying (1.5) once more, from Lemma 1 and (3.4)-(3.6) we obtain

$$
\begin{aligned}
& \int\left(\operatorname{Im} \kappa r+\frac{1}{4}-2 \epsilon\right) \frac{|\theta|^{2}}{\left(4+\log r / r_{0}\right)^{2}} d x \leq \frac{1}{4} C_{1} \int r^{2}\left(1+\log r / r_{0}\right)^{2}|f|^{2} d x \\
& +\frac{1}{4^{3} \epsilon}\left\{\int r^{2}|f|^{2} d x+\epsilon_{0}^{2} C_{1} \int r^{2}\left(1+\log r / r_{0}\right)^{2}|f|^{2} d x\right\} \\
& \leq \frac{1}{4}\left\{C_{1}+\frac{1}{4^{2} \epsilon}\left(1+\epsilon_{0}^{2} C_{1}\right)\right\} \int r^{2}\left(1+\log r / r_{0}\right)^{2}|f|^{2} d x
\end{aligned}
$$

which proves the inequality (1.7) if we choose $\epsilon=1 / 16$.

To proceed to the proof of Theorem 1(ii), we require one more lemma.

Lemma 4. For each $u \in H_{0, b}^{1}$ and $t>r_{0}$ we have

$$
\int_{\Omega_{t}} \frac{|u|^{2}}{4 r^{2}\left(1+\log r / r_{0}\right)^{2}} d x \leq \int_{\Omega_{t}}\left|\tilde{x} \cdot \nabla_{b} u\right|^{2} d x
$$


Proof. In the identity

$$
\begin{aligned}
\left|\tilde{x} \cdot \nabla_{b} u\right|^{2}= & \left|\tilde{x} \cdot \nabla_{b} u-\frac{u}{2 r\left(1+\log r / r_{0}\right)}\right|^{2}-\frac{|u|^{2}}{4 r^{2}\left(1+\log r / r_{0}\right)^{2}} \\
& +2 \operatorname{Re}\left\{\tilde{x} \cdot \nabla_{b} u \frac{\bar{u}}{2 r\left(1+\log r / r_{0}\right)}\right\},
\end{aligned}
$$

the last term on the right can be rewritten as

$$
\nabla \cdot\left\{\tilde{x} \frac{|u|^{2}}{2 r\left(1+\log r / r_{0}\right)}\right\}+\frac{|u|^{2}}{2 r^{2}\left(1+\log r / r_{0}\right)^{2}} .
$$

Integrate both sides over $\Omega_{t}$. Then the assertion follows from the identity

$$
\begin{aligned}
\int_{\Omega_{t}}\left|\tilde{x} \cdot \nabla_{b} u\right|^{2} d x= & \int_{\Omega_{t}}\left|\tilde{x} \cdot \nabla_{b} u-\frac{u}{2 r\left(1+\log r / r_{0}\right)}\right|^{2} d x \\
& +\int_{S_{t}} \frac{|u|^{2}}{2 r\left(1+\log r / r_{0}\right)} d S+\int_{\Omega_{t}} \frac{|u|^{2}}{4 r^{2}\left(1+\log r / r_{0}\right)^{2}} d x
\end{aligned}
$$

Remark 3. The above lemma gives a modification of the standard Hardy inequality

$$
\int \frac{|u|^{2}}{4 r^{2}\left(1+\log r / r_{0}\right)^{2}} d x \leq \int|\tilde{x} \cdot \nabla u|^{2} d x
$$

in an exterior domain.

Proof of Theorem 1(ii). We return to the identity of Proposition 1. By (A1),

$$
c(x) \geq \frac{-1}{4 r^{2}\left(1+\log r / r_{0}\right)^{2}} .
$$

Then from Lemma 4 we have

$$
\operatorname{Im} \kappa \int_{r_{0}}^{\infty} \mu d r \int_{\Omega_{r}}\left\{\left|\nabla_{b} u\right|^{2}+c(x)|u|^{2}+|\kappa u|^{2}\right\} d x \geq 0
$$

and

$$
\begin{aligned}
\frac{1}{2} \int\left\{\left(\operatorname{Im} \kappa \frac{\mu}{r}+\frac{\mu}{4 r^{2}}-\frac{\mu^{\prime}}{2 r}\right)|u|^{2}+\mu\left(\left|\nabla_{b} u\right|^{2}+|\kappa u|^{2}\right)\right\} d x & \\
& \leq \frac{1}{2} \int \mu|\theta|^{2} d x+\int_{r_{0}}^{\infty} \mu(t) d t \int_{\Omega_{t}}|f(x)||i \kappa u| d x .
\end{aligned}
$$

By condition (1.8) on $\mu(t)$, this inequality reduces to

$$
\frac{1}{2} \int \mu\left(\left|\nabla_{b} u\right|^{2}+|\kappa u|^{2}\right) d x \leq \frac{1}{2} C_{3} \int \frac{|\theta|^{2}}{\left(4+\log r / r_{0}\right)^{2}} d x+\|\mu\|_{L^{1}} \int|f||i \kappa u| d x .
$$


Thus, the desired inequality (1.9) is concluded if we note (1.7) and

$$
\|\mu\|_{L^{1}} \int|f||i \kappa u| d x \leq\|\mu\|_{L^{1}}^{2} \int \mu^{-1}|f|^{2} d x+\frac{1}{4} \int \mu|\kappa u|^{2} d x .
$$

\section{§4. Proof of Theorem 2}

The following proposition summarizes abstract results which allow us to employ the resolvent estimate for a selfadjoint operator to obtain a space-time weighted estimate for the associated evolution equation. For the proof of the proposition see, e.g., [14], [18].

Let $\Lambda$ be a selfadjoint operator in a Hilbert space $\mathcal{H}$, and for $z \in \mathbb{C} \backslash \mathbb{R}$ let $\mathcal{R}(z)$ be the resolvent of $\Lambda$. Suppose that $A$ is a densely defined, closed operator from $\mathcal{H}$ to another Hilbert space $\mathcal{H}_{1}$.

Proposition 4. Assume that there exists $C>0$ such that

$$
\sup _{z \notin \mathbb{R}}\left\|A \mathcal{R}(z) A^{*} f\right\|_{\mathcal{H}_{1}}<\sqrt{C}\|f\|_{\mathcal{H}_{1}}
$$

for $f \in \mathcal{D}\left(A^{*}\right)$. Then

$$
\begin{aligned}
\left|\int_{0}^{ \pm \infty}\left\|\int_{0}^{t} A e^{-i(t-\tau) \Lambda} A^{*} g(\tau) d \tau\right\|_{\mathcal{H}_{1}}^{2} d t\right| & \leq C\left|\int_{0}^{ \pm \infty}\|g(t)\|_{\mathcal{H}_{1}}^{2} d t\right|, \\
\sup _{t \in \mathbb{R}_{ \pm}}\left\|\int_{0}^{t} e^{i \tau \Lambda} A^{*} g(\tau) d \tau\right\|_{\mathcal{H}}^{2} & \leq 2 \sqrt{C}\left|\int_{0}^{ \pm \infty}\|g(t)\|_{\mathcal{H}_{1}}^{2} d t\right|
\end{aligned}
$$

for each $g \in L^{2}\left(\mathbb{R} ; \mathcal{D}\left(A^{*}\right)\right)$, and

$$
\left|\int_{0}^{ \pm \infty}\left\|A e^{-i t \Lambda} f\right\|_{\mathcal{H}_{1}}^{2} d t\right| \leq 2 \sqrt{C}\|f\|_{\mathcal{H}}^{2}
$$

for each $f \in \mathcal{H}$.

Proof of Theorem 2(i). Set $\Lambda=L, \mathcal{H}=\mathcal{H}_{1}=L^{2}$ and $A=r^{-1}\left(1+\log r / r_{0}\right)^{-1}$ (multiplication operator). Then $A^{*}=A$ and $\mathcal{R}(z)=R(z)$, and if we let $z=\kappa^{2}$, then it follows from Theorem 1(i) that

$$
\begin{aligned}
\left\|A R(z) A^{*} f\right\| & =\left\|r^{-1}\left(1+\log r / r_{0}\right)^{-1} R(z) A^{*} f\right\| \\
& \leq \sqrt{C_{1}}\left\|r\left(1+\log r / r_{0}\right) A^{*} f\right\|=\sqrt{C_{1}}\|f\| .
\end{aligned}
$$


Thus, estimates (4.2) and (4.3) hold, and can be written as

$$
\begin{aligned}
\left|\int _ { 0 } ^ { \pm \infty } \left\|r^{-1}\left(1+\log r / r_{0}\right)^{-1} \int_{0}^{t} e^{-i(t-\tau) L} h(\tau) d \tau\left|\|^{2} d t\right|\right.\right. & \leq C_{1}\left|\int_{0}^{ \pm \infty}\left\|r\left(1+\log r / r_{0}\right) h(t)\right\|^{2} d t\right|, \\
\left|\int_{0}^{ \pm \infty}\left\|r^{-1}\left(1+\log r / r_{0}\right)^{-1} e^{-i t L} f\right\|^{2} d t\right| & \leq 2 \sqrt{C_{1}}\|f\|^{2},
\end{aligned}
$$

where $h(t)=r^{-1}\left(\log \left(1+\log r / r_{0}\right)^{-1} g(t)\right.$ and $f \in L^{2}(\Omega)$. By the limit procedure the first inequality is continuously extended to $h(t)$ satisfying $r\left(1+\log r / r_{0}\right) h(t) \in$ $L^{2}(\mathbb{R} \times \Omega)$, as desired.

To show Theorem 2(ii) we consider the Klein-Gordon equation

$$
i \partial_{t} u=\Lambda u, \quad u(t)=\left\{w(t), \partial_{t} w(t)\right\}, \quad \Lambda=\left(\begin{array}{cc}
0 & i \\
-i\left(L+m^{2}\right) & 0
\end{array}\right)
$$

in the energy space $\mathcal{H}=\dot{H}_{b}^{1} \times L^{2}$, where $\dot{H}_{b}^{1}$ is the completion of $C_{0}^{\infty}(\Omega)$ in the norm

$$
\left\|f_{1}\right\|_{\dot{H}_{b}^{1}}^{2}=\frac{1}{2} \int\left\{\left|\nabla_{b} f_{1}\right|^{2}+\left(c(x)+m^{2}\right)\left|f_{1}\right|^{2}\right\} d x .
$$

Then $\Lambda$ with domain

$$
\mathcal{D}(\Lambda)=\left\{f_{1} \in \dot{H}_{b}^{1} ; \Delta_{b} f_{1} \in L^{2}\right\} \times\left\{f_{2} \in \dot{H}_{b}^{1} \cap L^{2}\right\}
$$

forms a selfadjoint operator in $\mathcal{H}$, and its resolvent is given by

$$
\mathcal{R}(z)=\left(L+m^{2}-z^{2}\right)^{-1}\left(\begin{array}{cc}
z & i \\
-i\left(L+m^{2}\right) & z
\end{array}\right) .
$$

Let $A: \mathcal{H} \rightarrow \mathcal{H}_{1}=L^{2}$ be defined by

$$
A f=r^{-1}\left(1+\log r / r_{0}\right)^{-1} \sqrt{L+m^{2}} f_{1} \quad \text { for } f=\left\{f_{1}, f_{2}\right\} \in \mathcal{H} .
$$

Then the adjoint operator $A^{*}$ is given by

$$
A^{*} g=\left\{2{\sqrt{L+m^{2}}}^{-1} r^{-1}\left(1+\log r / r_{0}\right)^{-1} g, 0\right\} \quad \text { for } g \in L^{2} \text {. }
$$

Proof of Theorem 2(ii). By definition

$$
\begin{aligned}
A \mathcal{R}(z) A^{*} g= & 2 r^{-1} \log \left(1+\log r / r_{0}\right)^{-1} \\
& \times z\left(L+m^{2}-z^{2}\right)^{-1} r^{-1}\left(1+\log r / r_{0}\right)^{-1} g
\end{aligned}
$$


for $g \in \mathcal{D}\left(A^{*}\right)$. Then since

$$
\begin{aligned}
\int \mid r^{-1}(1+ & \left.\log r / r_{0}\right)\left.^{-1} z\left(L+m^{2}-z^{2}\right)^{-1} f\right|^{2} d x \\
\leq & m^{2} \int r^{-2}\left(1+\log r / r_{0}\right)^{-2}\left|\left(L+m^{2}-z^{2}\right)^{-1} f\right|^{2} d x \\
& +\int r^{-2}\left(1+\log r / r_{0}\right)^{-2}\left|-m^{2}+z^{2}\right|\left|\left(L+m^{2}-z^{2}\right)^{-1} f\right|^{2} d x
\end{aligned}
$$

using (1.5) and (1.9) with $\mu=r^{-2}\left(1+\log r / r_{0}\right)^{-2}$ of Theorem 1 , we obtain

$$
\left\|A \mathcal{R}(z) A^{*} g\right\| \leq 2 \sqrt{m^{2} C_{1}+2 C_{4}}\|g\|
$$

We return to Proposition 4 with this inequality. Let $w(t)$ be the first component of the solution $e^{-i t \Lambda} f$. Then (4.4) shows that

$$
\begin{aligned}
\left|\int_{0}^{ \pm \infty}\left\|A e^{-i t \Lambda} f\right\|^{2} d t\right| & =\left|\int_{0}^{ \pm \infty}\left\|r^{-1}\left(1+\log r / r_{0}\right)^{-1} \sqrt{L+m^{2}} w(t)\right\|^{2} d t\right| \\
& \leq 4 \sqrt{m^{2} C_{1}+2 C_{4}}\|f\|_{\mathcal{H}}^{2} .
\end{aligned}
$$

Since

$$
w(t)=\cos \left(t{\sqrt{L+m^{2}}}^{2} f_{1}+{\sqrt{L+m^{2}}}^{-1} \sin \left(t \sqrt{L+m^{2}}\right) f_{2},\right.
$$

choosing $f=\left\{{\sqrt{L+m^{2}}}^{-1} g, 0\right\}$ and $f=\{0, g\}$ for $g \in L^{2}$, we obtain

$$
\left|\int_{0}^{ \pm \infty}\left\|r^{-1}\left(1+\log r / r_{0}\right)^{-1} \cos \left(t \sqrt{L+m^{2}}\right) g\right\|^{2} d t\right| \leq 2 \sqrt{m^{2} C_{1}+2 C_{4}}\|g\|^{2}
$$

and

$$
\left|\int_{0}^{ \pm \infty}\left\|r^{-1}\left(1+\log r / r_{0}\right)^{-1} \sin \left(t \sqrt{L+m^{2}}\right) g\right\|^{2} d t\right| \leq 2 \sqrt{m^{2} C_{1}+2 C_{4}}\|g\|^{2},
$$

respectively. These inequalities imply assertion (ii).

\section{Acknowledgements}

The first author was partially supported by Grant-in-Aid for Scientific Research (C) (No. 25400179), Japan Society for the Promotion of Science.

The second author was partially supported by Grant-in-Aid for Scientific Research (C) (No. 23540222), Japan Society for the Promotion of Science.

The authors would like to express their gratitude to the referees for carefully reading the manuscript and making valuable suggestions. 


\section{References}

[1] A. R. Aliev and E. H. Eyvazov, On the essential spectrum of electromagnetic Schrödinger operator with singular electric potential, Complex Var. Elliptic Equations 59 (2014), 18-27. Zbl 1284.35298 MR 3170737

[2] M. Ben-Artzi, Global estimates for the Schrödinger equation, J. Funct. Anal. 107 (1992), 362-368. Zbl 0774.35019 MR 1172030

[3] S. Cuccagna and P. P. Schirmer, On the wave equation with magnetic potential, Comm. Pure Appl. Math. 54 (2001), 135-152. Zbl 1032.35122 MR 1794351

[4] P. D'Ancona and L. Fanelli, Strichartz and smoothing estimates for dispersive equations with magnetic potentials, Comm. Partial Differential Equations 33 (2008), 1082-1112. Zbl 1160.35363 MR 2424390

[5] M. B. Erdoğan, M. Goldberg and W. Schlag, Strichartz and smoothing estimates for Schrödinger operators with large magnetic potentials in $\mathbb{R}^{3}$, J. Eur. Math. Soc. 10 (2008), 507-531. Zbl 1152.35021 MR 2390334

[6] V. Georgiev, A. Stefanov and M. Tarulli, Smoothing-Strichartz estimates for the Schrödinger equation with small magnetic potential, Discrete Contin. Dynam. Systems Ser. A 17 (2007), 771-786. Zbl 1125.35077 MR 2276474

[7] A. Jensen and G. Nencieu, A unified approach to resolvent expansions at thresholds, Rev. Math. Phys. 15 (2001), 717-754. Zbl 1029.81067 MR 1841744

[8] T. Kato, Wave operators and similarity for some non-selfadjoint operators, Math. Ann. 162 (1966), 258-279. Zbl 0139.31203 MR 0190801

[9] T. Kato and K. Yajima, Some examples of smooth operators and the associated smoothing effect, Rev. Math. Phys. 1 (1989), 481-496. MR 1061120

[10] T. Kobayashi and M. Misawa, $L^{2}$ boundedness for the $2 \mathrm{D}$ exterior problems for the semilinear heat and dissipative wave equations, in Harmonic analysis and nonlinear partial differential equations, RIMS Kôkyûroku Bessatsu B42, RIMS, Kyoto, 2013, 1-11. Zbl 1304.35155 MR 3220146

[11] S. T. Kuroda, An introduction to scattering theory, Lecture Notes Ser. 51, Aarhus Univ., 1978. Zbl 0407.47003 MR 0528757

[12] A. Laptev and T. Weidl, Hardy inequalities for magnetic Dirichlet forms, in Mathematical results in quantum mechanics (Prague, 1998), Oper. Theory Adv. Appl. 108, Birkhäuser, 1999, 299-305. Zbl 0977.26005 MR 1708811

[13] K. Mochizuki, Spectral and scattering theory for second order elliptic differential operators in an exterior domain, Lecture Notes Univ. Utah, Winter and Spring 1972.

[14] _ Uniform resolvent estimates for magnetic Schrödinger operators and smoothing effects for related evolution equations, Publ. RIMS Kyoto Univ. 46 (2010), 741-754. Zbl 05834704 MR 2791005

[15] _ Resolvent estimates for magnetic Schrödinger operators and their applications to related evolution equations, Rend. Istit. Mat. Univ. Trieste 42 (2010), suppl., 143-164. Zbl 1232.35141 MR 2760482

[16] M. Murata, Asymptotic expansions in time for solutions of Schrödinger-type equations, J. Funct. Anal. 49 (1982), 10-56. Zbl 0499.35019 MR 0680855

[17] H. Nakazawa, Uniform resolvent estimates for Schrödinger equations in an exterior domain in $\mathbb{R}^{2}$ and their applications to scattering problems, Bull. Liberal Arts Sci. Nippon Medical School 42 (2013), 1-12.

[18] M. Reed and B. Simon, Methods of modern mathematical physics. Vol. 3, Academic Press, San Diego, CA, 1979. Zbl 0405.47007 MR 0529429

[19] W. Schlag, Dispersive estimates for Schrödinger operators in dimension two, Comm. Math. Phys. 257 (2005), 87-117. Zbl 1134.35321 MR 2163570 
[20] K. Watanabe, Smooth perturbations of the selfadjoint operator $|\Delta|^{\alpha / 2}$, Tokyo J. Math. 14 (1991), 239-250. Zbl 0757.47015 MR 1108171

[21] D. R. Yafaev, Mathematical scattering theory. Analytic theory, Math. Surveys Monogr. 158, Amer. Math. Soc., Providence, RI, 2009. Zbl 1197.35006 MR 2598115

[22] K. Yajima, Schrödinger evolution equation with magnetic fields, J. Anal. Math. 56 (1991), 29-76. Zbl 0739.35083 MR 1243098

[23] $\_L^{p}$-boundedness of wave operators for two dimensional Schrödinger operators, Comm. Math. Phys. 208 (1999), 125-152. Zbl 0961.47004 MR 1729881 